\title{
Características físicas y antropométricas, y análisis de juego en jugadores de pádel de elite
}

\author{
Physical and anthropometric characteristics, and \\ match-analysis in elite padel players
}

\author{
Zabala-Lili J, ${ }^{1}$ Gil SM, ${ }^{1}$ Badiola A, ${ }^{1}$ Bidaurrazaga-Letona I, ${ }^{1}$ Vargas A, ${ }^{1}$ Granados $C,{ }^{1}$. \\ ${ }^{1}$ Universidad del País Vasco/Euskal Herriko Unibertsitatea, España. *Correspondencia: granados. \\ cristina@ehu.eus
}

Recibido: 10-02-2016; Aceptado: 07-03-2016

\section{Resumen.}

Los objetivos de este estudio son, por un lado, determinar las características antropométricas y de fuerza (medida mediante test de hand-grip y salto vertical con brazos libres) comprándolo con otros deportes de raqueta, y por otro, analizar las características propias del juego durante el Campeonato de España de Pádel sub-23. Para ello 14 hombres (20,38 $\pm 1,92$ años) y 12 mujeres (19,73 $\pm 2,37$ años) participaron en este estudio. A todos ellos se les realizó unas mediciones antropométricas, y unos tests de fuerza de miembro superior (hand-grip) y de miembro inferior (salto vertical con brazos libres) antes y después de los partidos. Por último, se registraron los datos de analisis de juego durante dicho evento. Los resultados muestran que las características antropométricas de los jugadores de pádel difieren ligeramente de las de los jugadores de otros deportes de raqueta. Los valores de fuerza, no disminuyeron después del partido, no observándose ningún indicio de fatiga. Respecto al análisis de las características del juego, el pádel se caracteriza por tener menos tiempo activo que el tenis de mesa y el badminton, pero más tiempo activo que el tenis.

Palabras clave: Antropometría, fuerza, análisis del juego, padel, élite. 


\section{Abstract.}

The goals of the present study were, on the one hand, to determine the anthropometric, strength (measured by jump and hand-grip tests) compared to other racquet sports, and on the other hand, to analyze the time-motion characteristics during the under-23 Spanish Paddle championships. Thus, 14 men $(20,38 \pm 1,92 \mathrm{yr})$ and 12 women $(19.73 \pm 2,37 \mathrm{yr})$ participated in this study. Firstly, participants were assessed for anthropometric measurements; secondly, strength was measured by jump and hand-grip tests before and immediately after the match; finally, the time-motion of matches were quantified during the under-23 Spanish Championship in 2012. The anthropometric characteristics of paddle players were slightly different in comparison to players of other racket sports. In respect of strength parameters, there were not significant differences between pre-match and post-match measurements, indicating no fatigue component after competition. In respect to time motion analyses, resting periods were significantly longer compared to the effective playing time. Paddle seems to have less time active than table-tennis and badminton, but more time active than tennis.

Key words: Anthropometry, strength, time-motion, paddle, elite

\section{Introducción}

El pádel es un deporte de reciente creación que ha tenido un crecimiento importante en los últimos años (Amieba y Salinero, 2013). Al igual que en otros deportes de raqueta, en el pádel, la actividad física implica esfuerzos de alta intensidad como sprints, saltos y golpeos agresivos de la pelota, intercalados con periodos de baja o nula intensidad (Martin, 2013). En las investigaciones precedentes se han analizado las características antropométricas, fisiológicas y de juego en otros deportes de raqueta como el tenis (Christmass et al., 1998; Ferrauti et al., 2001; Méndez-Villanueva et al., 2007; Méndez-Villanueva et al., 2010; Kovacs, 2007; Girard y Millet, 2009; Fernández-Fernández et al., 2007; Fernández-Fernández et al., 2008; Fernández-Fernández et al., 2009), el badminton (Faude et al., 2007; Cabello y González-Badillo, 2003; Torres et al., 2004), el squash (Wells et al., 2004; Vuckovic et al., 2004; Girard et al., 2007) o el tenis de mesa (Zagatto et al., 2010), dichas investigaciones han contribuido al desarrollo del juego y a concretar las necesidades específicas de cada uno de ellos.
A pesar de que el pádel ha aumentado en popularidad en las últimas décadas y se puede encontrar algún trabajo sobre las características antropométricas de los jugadores del pádel (Martínez-Rodríguez et al., 2014), para el conocimiento de los autores del presente artículo hay muy pocos estudios que analicen de manera conjunta aspectos físicos y de análisis de juego en el pádel. En el estudio realizado por Amieba y Salinero (2013), se analizan los aspectos generales de la competición del pádel y sus demandas fisiológicas en ocho jugadores amateur simulando un partido de pádel. Sin embargo, no hay ningún estudio que analice un campeonato oficial en jugadores de élite. El conocimiento científico de estos aspectos posibilitaría conocer los requerimientos concretos de los jugadores de pádel, y así mejorar todo el proceso de entrenamiento.

Por ello, los objetivos de este estudio son, por un lado, determinar las características antropométricas y de fuerza (medida mediante test de handgrip y salto vertical con brazos libres) en los jugadores de pádel comparándolo con otros deportes de raqueta, y por otro, analizar ciertas características propias del juego durante el Campeonato de España de Pádel sub-23. 


\section{Metodología}

Participantes. Veintiséis jugadores de pádel (masculinos $n=14$, edad: 20,38 $\pm 1,92$ años; femeninos $n=12$, edad: $19,73 \pm 2,37$ años) participaron en este estudio durante el Campeonato de España de Pádel sub-23. Todos ellos tenían una amplia experiencia de entrenamiento $(8,07 \pm 3,75$ años $) y$ fueron considerados jugadores de élite porque en diferentes ocasiones habían ganado varios campeonatos de nivel estatal y/o mundial.

Todos los jugadores firmaron un consentimiento informado antes de su participación en el estudio. Previamente se les informó, tanto oral como por escrito, sobre los riesgos potenciales y beneficios resultantes de su participación en el mismo, tal y como se concreta en la Declaración de Helsinki (2008). Los participantes eran libres de abandonar el estudio voluntariamente en cualquier momento.

Diseño Experimental. Las mediciones fueron realizadas durante el Campeonato de España de Pádel sub-23 en julio de 2012. Este torneo se organizó en rondas clasificatorias, de modo que la pareja ganadora se clasificaba para la siguiente ronda y jugaba otra eliminatoria.

A todos los participantes se les tomaron las medidas antropométricas antes de la disputa del primer partido del campeonato. Los test de fuerza (handgrip y salto vertical con brazos libres) fueron realizados antes de los partidos (tras un calentamiento no estandarizado de 15 minutos) e inmediatamente después de acabarlos. Todos los partidos fueron grabados en vídeo para el análisis posterior de las características propias del juego.

Procedimientos. Todos los participantes fueron cuidadosamente informados sobre la ejecución de los test y estos se realizaron en el mismo orden en todos los casos.
Características antropométricas. La talla $(\mathrm{cm})$ y la masa corporal $(\mathrm{kg})$ fueron medidas utilizando un estadiómetro de precisión y una balanza (Seca, Alemania), posteriormente se calculó el índice de masa corporal (IMC, kg/m $\left.{ }^{2}\right)$. Los perímetros del brazo relajado y contraído, muslo y pantorrilla fueron medidos usando una cinta métrica flexible (W606PM, Lufkin, Alemania); los diámetros del húmero biepicondíleo (codo), biestiloideo (muñeca), femur biepicondíleo (rodilla) y bimaleolar (tobillo), la longitud de la mano y la anchura de los hombros fueron medidos con un calibre (Rosscraft, Canadá).

Los seis pliegues cutáneos (tríceps, subscapular, abdominal, suprailíaco, muslo anterior y pantorrilla media) fueron medidos con la ayuda de un plicómetro (Holtain, Crymych, Reino Unido), y se calculó la suma de todos los pliegues, la composición corporal (Yuhasz, 1974) y el somatotipo (Heath and Carter, 1967) para cada jugador.

Mediciones de fuerza. El test de salto fue realizado en una pista cubierta de pádel y consistió en dos saltos verticales máximos en contramovimiento con brazos libres sobre una plataforma de contacto (Microgate Optojump, Italia) con aproximadamente 10 segundos de descanso entre ellos. Los participantes partían de una posición recta, y realizaban un movimiento preparatorio desde esa posición hasta $90^{\circ}$ de flexión en las rodillas, con la subsiguiente acción concéntrica máxima. Los participantes podían mover los brazos libremente durante el salto, pero tenían que aterrizar en la plataforma de contacto en una posición similar a la del despegue. La distancia de salto se calculó a partir del tiempo de vuelo (Bosco et al, 1983). Para los posteriores análisis, se usó el mejor salto obtenido, y los resultados fueron relativizados en función de la masa corporal $(\mathrm{cm} / \mathrm{kg})$ y del porcentaje de masa muscular (cm/\%MM) de cada jugador. 
La fuerza de la mano dominante fue medida mediante el test de hand-grip (Fallahi y Jadidian, 2011). El test fue realizado en posición sentada y con el brazo totalmente extendido. Se registró la fuerza $(\mathrm{kg})$ mediante un dinamómetro manual portátil (Jamar, EEUU). Los participantes ejecutaron tres intentos, realizando una contracción isométrica máxima de 5 segundos cada uno de ellos, con un descanso de al menos un minuto entre repeticiones. Se les indicó que debían apretar el dinamómetro tan fuerte como les fuera posible. Para los posteriores análisis, se usó el mejor valor obtenido, y los resultados fueron valorados en función de la masa corporal $(\mathrm{kg} / \mathrm{kg})$ y del porcentaje de masa muscular (kg/\%MM) de cada jugador.

Análisis del juego. El análisis de las características del juego del pádel se desarrolló mediante la filmación de los partidos durante el campeonato. En el presente estudio se filmaron las semifinales del torneo femenino y las finales de los torneos femenino y masculino. La grabación fue llevada a cabo con una cámara fija en el centro de la pista a $5 \mathrm{~m}$ de altura. Las grabaciones fueron analizadas utilizando un ordenador portátil y un software especializado (Cyberlink Power Director 11, Taiwan), para establecer al detalle los tiempos de juego y pausa. Esto posibilitó calcular las siguientes variables: a) la duración de los tantos (DT, segundos); b) tiempo total de juego (TTJ, segundos); c) tiempo real de juego (TRJ, segundos); d) tiempo efectivo de juego (TEJ, porcentaje de TRJ respecto TTJ, \%); e) densidad de trabajo (DW, ratio TRJ-tiempo de pausa, variable no dimensional); f) ratio trabajo-pausa (W-P r, duración del tiempo de pausa respecto a cada segundo de juego). Además, se cuantificaron el número de tantos ( $T$, total tantos) y puntos (TP, total puntos) que ocurrían en cada juego. El análisis del juego se realizó por el mismo investigador en todos los casos.
Estadística. El tratamiento estadístico se realizó utilizándose el programa de análisis de Ciencias Sociales SPSS 20.0 (StatSoft, Tulsa, OK, USA). Se realizó una estadística descriptiva presentándose los valores como media \pm desviación estándar (DE). Se comprobó la normalidad de los datos en cada una de las variables mediante la prueba de Shapiro-Wilk y la homogeneidad de la varianzas mediante el test de Levene. Para determinar las diferencias antes y después del partido se realizó la prueba de T-student para muestras relacionadas. Cuando las variables no eran distribuidas normalmente, se optó el test de Wilcoxon. Para el análisis estadístico se utilizó el mejor valor de cada test. El nivel de significación para todos los test se estableció en $p<0,05$.

\section{Resultados}

Características antropométricas. Las características antropométricas principales de los participantes se muestran en la tabla 1.

Mediciones de fuerza. En la tabla 2 se pueden apreciar los valores de fuerza, absolutos y relativizados respecto a la masa corporal y al \%MM, obtenidos por los jugadores de pádel masculinos y femeninos, en las pruebas de salto vertical con brazos libres y de hand-grip. En ambos grupos, no se observaron diferencias significativas entre las mediciones previas y posteriores a los partidos.

Análisis del juego. Los resultados del análisis del juego de los partidos se muestran en la tabla 3. La media de la DT de los cuatro partidos analizados fue de 9,02 \pm 8,19 segundos y no se observaron diferencias significativas entre ninguno de los cuatro partidos. El TT] de los partidos no fue homogéneo, pero en todos ellos el periodo activo tuvo una duración menor que el tiempo de pausa, siendo el TEJ el 38\% del TTJ. 
Zabala et al. Características físicas y antropométricas y análisis de juego

Tabla l. Parámetros antropométricos principales de los jugadores de pádel.

\begin{tabular}{|c|c|c|}
\hline & Masculinos (n=14) & Femeninos (n=12) \\
\hline Edad (años) & $20,38(1,92)$ & $19,73(2,37)$ \\
\hline Talla (cm) & $178,89(7,43)$ & $166,82(7,43)$ \\
\hline Masa (kg) & $74,27(11,35)$ & $58,06(6,76)$ \\
\hline $\operatorname{IMC}\left(\mathrm{kg} / \mathrm{m}^{2}\right)$ & $23,12(2,49)$ & $20,84(1,50)$ \\
\hline Longitud mano $(\mathrm{cm})$ & $18,95(1,11)$ & $17,53(0,96)$ \\
\hline$\sum 6$ pliegues $(\mathrm{mm})$ & $76,45(19,49)$ & $94,79(24,67)$ \\
\hline$\sum$ pliegues extr. (mm) & $39,14(8,74)$ & $53,18(11,00)$ \\
\hline$\sum$ pliegues tronco $(m m)$ & $37,30(14,85)$ & $41,60(16,09)$ \\
\hline Brazo relajado $(\mathrm{cm})$ & $29,57(2,19)$ & $25,50(1,36)$ \\
\hline Brazo (cm) & $31,94(2,24)$ & $26,66(1,48)$ \\
\hline$\% \mathrm{MG}$ & $13,32(2,54)$ & $18,25(3,81)$ \\
\hline$\% \mathrm{MO}$ & $17,03(1,66)$ & $16,77(1,23)$ \\
\hline$\% \mathrm{MM}$ & $45,53(1,97)$ & $40,86(3,15)$ \\
\hline Endomorfia & $2,96(0,83)$ & $3,89(1,38)$ \\
\hline Mesomorfia & $6,99(1,26)$ & $3,29(0,84)$ \\
\hline Ectomorfia & $2,67(1,15)$ & $2,99(1,03)$ \\
\hline
\end{tabular}

$n$, número de participantes; IMC, indice de masa corporal; $\Sigma 6$ pliegues, (tríceps, subescapular, abdominal, suprailiaco, muslo anterior y pantorilla media); $\Sigma$ Pliegues extr., (triceps, muslo anterior y pantorilla media;

$\sum$ Pliegues tronco, (subescapular, abdominaly suprailiaco); \%MG, porcentaje de masa grasa; \%MO, porcentaje de masa ósea; \%MM, porcentaje de masa muscular. Los resultados son medias $( \pm \mathrm{DE})$.
Tabla2. Resultados descripirios de los tests de fuerza de los jugadores de pádel.

\begin{tabular}{|c|c|c|c|}
\hline & & 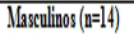 & Femeninos (n=12) \\
\hline \multicolumn{4}{|c|}{$\overline{S Y B L}$} \\
\hline & Absolutos $(\mathrm{cm})$ & $44,87(5,68)$ & $32,68(3,68)$ \\
\hline \multirow[t]{3}{*}{ PRE } & Relativos $(\mathrm{cm} / \mathrm{kg})$ & $0,60(0,12)$ & $0,58(0,09)$ \\
\hline & Relativos (cm/a.MM) & $0,99(0,13)$ & $0,80(0,08)$ \\
\hline & Absolutos (cm) & $45,23(6,24)$ & $34,07(5,14)$ \\
\hline \multirow[t]{4}{*}{ POST } & Realitivos (cm MC) & $0,63(0,13)$ & $0,59(0,10)$ \\
\hline & Relativos (cm/a.MM) & $1,00(0,14)$ & $0,84(0,13)$ \\
\hline & Hand-ging & & \\
\hline & Absolutus ( $(\mathrm{kg})$ & $55,50(8,82)$ & $34,67(4,80)$ \\
\hline \multirow[t]{3}{*}{ PRE } & Relativos (kg $\mathrm{kg})$ & $0,74(0,12)$ & $0,61(0,08)$ \\
\hline & Relativos (cm\%aMM) & $1,22(0,22)$ & $0,85(0,13)$ \\
\hline & Absolutos ( $(2 \mathrm{~g})$ & $33,91(9,41)$ & $35,50(5,56)$ \\
\hline & Relativos (kg $\mathrm{kg})$ & $0,73(0,11)$ & $0,61(0,11)$ \\
\hline & Relativos (cm\%MM) & $1,20(0,24)$ & $0,87(0,14)$ \\
\hline
\end{tabular}

STBL, test de salto vertical con brazos blores; PRE, mediciones precrias a p partido; POST, mediciones postenores a partido. Los resultados son medias $( \pm \mathrm{DE})$.

Tabla 3. Características del juego en el Campeonato de España sub-23 de pádel.

\begin{tabular}{lccccc}
\hline \hline Partido & Semi F1 & Semi F2 & Final F & Final M & Media (DE) \\
\hline \hline DT (s) & $8,76(8,15)$ & $8,19(7,87)$ & $10,01(9,63)$ & $8,92(7,40)$ & $9,02(8,19)$ \\
TTJ (s) & 3413 & 1753 & 3982 & 6431 & $3874,75(1937,29)$ \\
TRJ (s) & 1317,55 & 758,53 & 1381,94 & 2212,1 & $1417,53(599,13)$ \\
TEJ (\%) & 38,60 & 43,27 & 34,70 & 34,40 & $37,74(4,15)$ \\
DW & 0,63 & 0,76 & 0,53 & 0,52 & $0,57(0,11)$ \\
W-P r & $1: 1,60$ & $1: 1,31$ & $1: 1,88$ & $1: 1,91$ & $1: 1,67(0,28)$ \\
TP & 18 & 13 & 20 & 31 & $20,5(7,59)$ \\
TT & 102 & 86 & 117 & 208 & $128,25(54,65)$ \\
\hline \hline
\end{tabular}

Semi F1, primera semifinal, jugada por femeninas; Semi F2, segunda semifinal, jugada por femeninas; Final F, final jugada por femeninas; Final M, final jugada por masculinos; DT, duración de tantos (s); TTJ, tiempo total de juego (s); TRJ, tiempo real de juego (s); TEJ, tiempo efectivo de juego (\%); DW, densidad de trabajo; W-P r, ratio trabajo-pausa; TP, total puntos; TT, total tantos. Los resultados son la suma del tiempo y medias ( \pm DE).

La DT $(9,02 \pm 8,19$ s) y el TEJ $(37,74$ $\pm 4,15 \%)$ establecen una DW de 0,57 $\pm 0,11$ y un W-P r de $1: 1,67$, lo que significa que cada jugador descansa $1,67 \pm 0,28$ segundos por cada segundo activo de juego.

\section{Discusión}

Según nuestro conocimiento, este es el primer estudio que analiza las características físicas y de análisis de juego de manera simultánea en jugadores de pádel de élite en competición. Los jugadores masculinos de pádel del presente estudio, en comparación con deportistas de otros deportes de raqueta, fueron más altos y pesados que los jugadores de bádminton (Cabello y González-Badillo, 2003; Faude et al., 2007), y mostraron porcentajes de grasa corporal y un componente mesomórfico más alto que los jugadores de ténis de élite (Bergeron eta al., 1991; Bergeron et 
al., 1995; Kovacs, 2007; SánchezMuñoz et al., 2007). Respecto a las jugadoras femeninas, éstas presentaron una talla y masa similar a las jugadoras de bádminton de élite (Faude et al., 2007), pero un componente mesomórfico menor que las jugadoras de tenis de élite (Sánchez-Muñoz et al., 2007).

En lo que se refiere a las características de fuerza, los jugadores masculinos presentaron valores de fuerza explosiva de salto un $28 \%$ superiores que los jugadores de tenis (Girard y Millet, 2009). En lo que respecta a la fuerza máxima en el miembro superior, hay muy pocos estudios en deportistas de raqueta que la hayan evaluado mediante el test de handgrip. Pero si se comparan estos datos con los datos de la población en general aportados por Kolei y Pal Kaur (2011), las jugadoras del presente estudio mostraron unos valores $30 \%$ superiores a los presentados por la población en general femenina. Por tanto, estos resultados sugieren que los jugadores masculinos de pádel podrían ser más explosivos que los jugadores de tenis en las extremidades inferiores, y las femeninas más fuertes que la población general en las extremidades superiores. Por otro lado, los parámetros de fuerza no presentaron ninguna variación significativa entre las mediciones realizadas antes y después del partido, indicando que la fatiga no parece afectar al rendimiento a los jugadores de pádel de élite durante el transcurso de un partido. Este hecho también ha sido observado en el tenis, en el que los parámetros de fuerza explosiva se mantienen constantes a lo largo de un partido, excepto cuando la duración de este es superior a las 3 horas (Girard et al., 2006).

Las características de juego, como el ratio de trabajo-pausa y los porcentajes de los periodos activos y de pausa, han sido estudiadas ampliamente en los deportes de raqueta. Los valores medios de la duración de los tantos del presente estudio $(9,0 \pm 8,1 \mathrm{~s})$ son más largos que los observados en jugadores masculinos de tenis $(7,5 \pm 8,2 \mathrm{~s})$, de tenis de mesa
$(3,4 \pm 1,7 \mathrm{~s})$ y de bádminton $(5,5 \pm 4,0$ s), pero un $50 \%$ más reducidos que los valores observados en los jugadores de squash (Girard et al., 2007). El tiempo efectivo de juego fue un $38 \%$ del tiempo total de juego, 13-17\% más largo que en el caso del tenis (Fernández-Fernández et al., 2007; Fernández-Fernández et al., 2008; Méndez-Villanueva et al., 2007; Kovacs, 2007), pero un 2-12\% más corto que lo analizado en el tenis de mesa y el bádminton (Zagatto et al., 2010; Faude et al., 2007; Cabello y González-Badillo, 2003). En contraste, el tiempo efectivo de juego observado en el pádel es muy similar al observado en los partidos de squash (Girard et al., 2007). Todos estos estudios sugieren que el ratio de trabajopausa no es ni homogéneo, ni estable en los diferentes deportes de raqueta; sin embargo, este ratio está considerado como una variable objetiva para cuantificar la carga fisiológica de una actividad de raqueta (Duthie et al., 2003), y válido para desarrollar entrenamientos interválicos de manera individualizada en este tipo de deportistas (Fernandez-Fernandez et al., 2007). Por lo tanto, para poder describir estos aspectos más detalladamente, se considera necesario seguir investigando en esta área en estudios futuros.

\section{Conclusiones}

Respecto a las características antropométricas, los jugadores de pádel masculinos fueron más altos y pesados que los jugadores de bádminton (Cabello and González-Badillo, 2003; Faude et al., 2007), y mostraron mayor porcentaje graso y componente mesomórfico que los jugadores de tenis de élite (Bergeron et al., 1991; Bergeron et al., 1995; Kovacs, 2007; Sánchez-Muñoz et al., 2007). Las jugadoras femeninas, sin embargo, mostraron una talla y masa similar a las jugadoras de bádminton (Faude et al., 2007), pero un componente mesomórfico menor que sus homólogas del tenis (Sánchez-Muñoz et al., 2007).

En cuanto a los parámetros de fuerza, las jugadoras se mostraron más fuertes 
en miembros superiores que la población general, y los jugadores masculinos más explosivos que los jugadores de tenis de élite. No hubo diferencias significativas entre las mediciones de fuerza antes y después del partido, no observándose indicios de fatiga a lo largo de la competición.
Respecto a las características del juego, como ocurre en otros deportes de raqueta, los periodos de pausa fueron significativamente más largos que los periodos activos. El tiempo efectivo de juego es mayor en el pádel que en el tenis, pero menor que en el bádminton y el tenis de mesa, y muy similar al squash.

\section{Referencias}

Amieba, C., Salinero, J.J. (2013). Overview of paddle competition and its physiological demands. AGON. Int J Sport Sci, 3(2):60-67.

Bergeron, M.F., Maresh, C.M., Kraemer, W.J. (1991). Tennis: a physiological profile during match play. Int J Sports Med, 12(5):474-479.

Bergeron, M.F., Maresh, C.M., Amstrong, LE. (1995). Fluid-electrolyte balance associated with tennis match play in a hot environment. Int J Sports Nutr, 5:180-193.

Bosco, C., Luhtanen, P., Komi, P.V. (1983). A simple method for measurement of mechanical power in jumping. Eur J Appl Physiol, 50:273-282.

Cabello Manrique, D., González-Badillo, J.J. (2003). Analysis of the characteristics of competitive badminton. Brit J Sport Med, 37:62-66.

Christmass, M.A., Richmond, S.E., Cable, N.T., Arthur, P.G., Hartmann, P.E. (1998). Exercise intensity and metabolic response in singles tennis. J Sport Sci, 16:739-747.

Duthie, G., Pyne, D., Hooper, S. (2003). Applied physiology and game analysis of rugby union. Sports Med, 13:973991.
Fallahi, A.A., Jadidian, A.A. (2011). The effect of hand dimensions. Hand shape and some anthropometric characteristics on handgrip strength in male grip athletes and non-athletes. $J$ Hum Kinet, 29:151-159.

Faude, O., Meyer, T., Rosenberger, F., Fries, M., Huber, G., Kindermann, W. (2007). Physiological characteristics of badminton match play. Eur J Appl Physiol, 100:479-485.

Fernández-Fernández, J., MéndezVillanueva, A., Fernández-García, B., Terrados, N. (2007). Match activity and physiological responses during a junior female singles tennis tournament. Brit J Sport Med, 41:711716.

Fernández-Fernández, J., Sánz-Rivas, J., Fernández-García, B., MéndezVillanueva, A. (2008). Match activity and physiological load during a clay-court tennis tournament in elite female players. J Sport Sci, 26(14):1589-1595.

Fernández-Fernández, J., Sanz-Rivas, D., Méndez-Villanueva, A. (2009). A review of the activity profile and physiological demands of tennis match play. Strength Cond J, 31(4):15-26. 
Ferrauti, A., Bergeron, M.F., Pluim, B.M., Weber, K. (2001). Physiological responses in tennis and running with similar oxygen uptake. Eur $\mathrm{J} \mathrm{Appl}$ Physiol, 85:27-33.

Girard, O., Lattier, G., Micallef, J.P., Millet, G.P. (2006). Changes in exercise characteristics, maximal voluntary contraction, and explosive strength during prolonged tennis playing. Brit J Sport Med, 40(6):521-526.

Girard, O., Chevalier, R., Habrard, M., Sciberras, P., Hot, P., Millet, G.P. (2007). Game analysis and energy requirements of elite squash. $J$ Strength Cond Res, 21(3):909-914.

Girard, O., Millet, G. (2009). Physical determinants of tennis performance in competitive teenage players. $J$ Strength Cond Res, 23(6):18671872.

Heath, B.H., Carter, J.E.L. (1967). A modified somatotype method. Am J Phys Anthropol, 27:57-74.

Kolei, S., Pal Kaur, S. (2011). Correlations of handgrip strength with selected hand-arm-anthropometric variables in Indian inter-university female volleyball players. Asian J Sport Med, 2(4):220-226.

Kovacs, M.S. (2007). Tennis physiology: training the competitive athlete. Sports Med, 37(3):189-198.

Martin, R. (2013). Todo sobre el pádel. http://padelstar.es/preparacionfisica-padel/el-padel-es-un-deporteintermitente/.html.

Martínez-Rodríguez A., Roche Collado E., Vicente-Salar N. (2014). Body composition assessment of paddle and tennis adult male players. Nutr Hosp, 31(3):1294-301.

Méndez-Villanueva, A., FernándezFernández, J., Bishop, D., Fernández-
García, B., Terrados, N. (2007). Activity patterns, blood lactate concentrations and ratings of perceived exertion during a professional singles tennis tournament. Brit J Sport Med, 41:296-300.

Méndez-Villanueva, A., FernándezFernández, J., Bishop, D., FernándezGarcía, B. (2010). Ratings of perceived exertion-lactate association during actual singles tennis match play. J Strength Cond Res, 24:165-170.

Sánchez-muñoz, C., Sanz, D., Zabala, M. (2007). Anthropometric characteristics, body composition and somatotype of elite junior tennis players. Brit J Sport Med, 41(11):793799.

Torres, G., Cabello, D., Carrasco, L. (2004). Functional differences between tennis and badminton in young sportsmen. In: Science and racket sports III. Lees A, Khan JF, Maynard IW, eds. Oxon: Routledge, 185-189.

Vuckovic, G., Dezman, B., Erculj, F., Kovacic, S., Pers, J. (2004). Differences between the winning and the losing players in a squash game in terms of distance covered. In: Science and racket sports III. Lees A, Khan JF, Maynard IW, eds. Oxon: Routledge, 202-207.

Wells, J., Robertson, C., Hughes, M., Howe, D. (2004). Performance profiles of elite men squash doubles match play. In: Science and Racket Sports III. Lees A, Khan JF, Maynard IW, eds. Oxon: Routledge, 196-201.

Yuhasz, M.S. (1974). Physical fitness Manual. London. Ontario: University of Western Ontario.

Zagatto, A.M., Morel, E.A., Gobatto, C.A. (2010). Physiological responses and characteristics of table tennis matches determined in official tournaments. J Strength Cond Res, 24(4):942-949. 